\title{
Low-dose Pressurized Intrathoracic Aerosol Chemotherapy (PITAC) as an Alternative Therapy for Pleuropulmonary Involvement in Pseudomyxoma Peritonei
}

\author{
MANUELA ROBELLA ${ }^{1}$, MARCO VAIRA ${ }^{1}$, ALICE BORSANO ${ }^{1}$, \\ CLAUDIO MOSSETTI ${ }^{2}$ and MICHELE DE SIMONE ${ }^{1}$ \\ ${ }^{1}$ Unit of Surgical Oncology, Candiolo Cancer Institute, IRCCS - FPO, Candiolo, Italy; \\ ${ }^{2}$ Department of Thoracic Surgery, AOU Città della Salute e della Scienza di Torino - University of Torino, Turin, Italy
}

\begin{abstract}
Background/Aim: Pseudomyxoma peritonei (PMP) is a rare disease characterized by mucinous ascites and widespread peritoneal implants. It usually originates from the rupture of an adenoma/adenocarcinoma of the appendix. Although this tumor is only superficially invasive and does not metastasize, it could be a fatal disease. Extra-abdominal spread of PMP is an unusual occurrence with few reports in medical literature. Case Report: A 50-year-old man was diagnosed with PMP according to the findings of thorax and abdomen CT scan and cytologic and histological examinations. The radiological exam showed irregular thickening on the surface of left diaphragmatic and parietal pleura. Results: First, cytoreductive surgery associated with hyperthermic intraperitoneal chemotherapy (HIPEC) for the abdominal disease was performed. Histopathological examination confirmed the diagnosis of low grade PMP. The radiological evaluation performed 5 months later showed a dimensional increase in pleural nodules. The treatment consisted of an extensive intrathoracic cytoreductive surgery in combination with pressurized intra-thoracic aerosol chemotherapy (PITAC). Postoperative course was uneventful. Conclusion: PMP with pleural extension is a rare phenomenon and carries an unfavourable prognosis. Due to the rarity of this presentation, its correct treatment is still unclear. We present a therapeutic approach to be applied in selected patients.
\end{abstract}

Pseudomyxoma peritonei (PMP) is a rare disease that affects around two people of all ages per million each year, both men

Correspondence to: Manuela Robella, Unit of Surgical Oncology, Candiolo Cancer Institute, IRCCS - FPO Strada Provinciale 142, km 3.95, 10060 Candiolo (TO), Italy. Tel: +39 0119933630, +39 3383824104,e-mail: manuela.robella@ircc.it

Key Words: Pseudomyxoma Peritonei, locoregional chemotherapy, PITAC. and women. It's characterised by big amount of mucinous ascites and low and progressive accumulations of implants on the peritoneal surfaces. PMP usually originates from a mucinous appendiceal tumor; more rarely, it can start in other parts of the body such as the bowel, an ovary or the bladder.

PMP symptoms usually take a while to appear and vary widely among patients; the most common are abdominal swelling or an increase in abdominal girth, changes in bowel habit, general fatigue and new-onset hernia.

Pleural extension from PMP is unusual and seems to be related to congenital pleuroperitoneal communication, diaphragmatic perforation during surgery, transdiaphragmatic spreading through lymphatic lacunae and, rarely, through direct invasion of the diaphragm (1-7).

The successful treatment of abdominal PMP required the combined approach including cytoreductive surgery associated with hyperthermic intraperitoneal chemotherapy (HIPEC). Debated recommendations for the management of pleural extension consist of surgical cytoreduction and hyperthermic intrathoracic chemotherapy $(\operatorname{HITOC})(7,8)$.

We report a case of a 50-year-old man with PMP and intrathoracic involvement who underwent first cytoreductive surgery and HIPEC for the abdominal disease and secondly pleurectomy, decortication and PITAC for the pleural disease.

\section{Case Report}

A 50-year-old man with a history of abdominal PMP was referred to our centre after three cycles of neoadjuvant chemotherapy with Folfox schedule. Physical examination showed a distended abdomen. Complete blood analysis was within normal levels, carcinoembryonic antigen (CEA) and cancer antigen 125 (CA-125) levels slightly increased, 6.8 $\mathrm{ng} / \mathrm{ml}$ and $63.8 \mathrm{U} / \mathrm{ml}$, respectively.

Computed tomography revealed significant mucinous ascites, omental cake, widespread peritoneal carcinomatosis and left pleural effusion. He was submitted to an explorative 

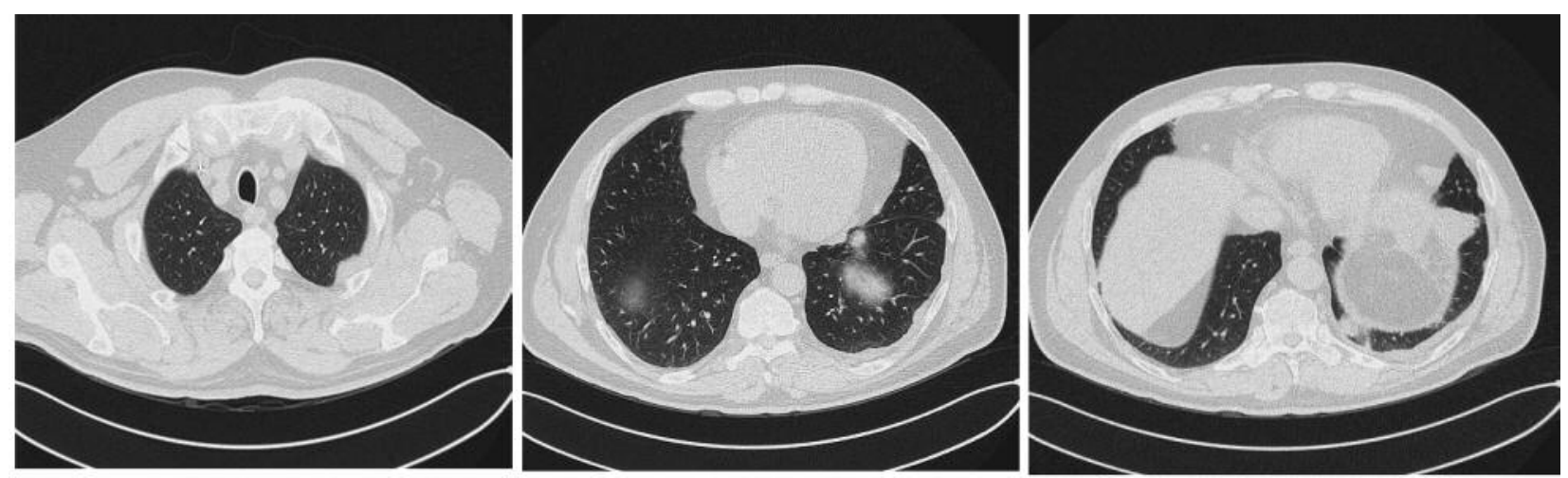

Figure 1. Chest CT scan showing left pleural disease.

laparoscopy which revealed massive gelatinous ascites and a laparoscopic peritoneal cancer index (PCI) of 37. Biopsies confirmed the diagnosis of intermediate PMP according to Ronnett classification (9) and low grade PMP according to WHO Classification (10).

The patient underwent extensive cytoreductive surgery: total peritonectomy (including diaphragmatic peritoneum), omentectomy, splenectomy, hepatic Glisson's capsule resection, cholecystectomy, lesser omentum resection, small bowel resection, right colectomy and anterior rectal resection. No injury was reported to the diaphragms. Treatment was completed by HIPEC with cisplatin $100 \mathrm{mg} / \mathrm{sm}$ and mytomicin C $16 \mathrm{mg} / \mathrm{sm}$ for 60 minutes at a temperature of $41.5^{\circ} \mathrm{C}$ according to the original semiclosed HIPEC technique (11). The operation was then completed with the creation of anastomoses and a temporary loop ileostomy.

Hystopathological examination confirmed the diagnosis of PMCA with intermediate features (PMCA-I/D) or low grade PMP.

Imaging evaluation five months after surgery, before ileostomy closure, revealed left pleural effusion, dimensional increase of left pleural nodules (Figure 1). The diagnosis was reached with percutaneous thoracic biopsy and finding of mucinous malignant cells.

The clinical case was discussed in the interdisciplinary tumor board: surgery associated with locoregional chemotherapy was indicated. Patient was extensively informed about the procedure and signed an informed consent.

Left thoracotomy was accompanied by a resection of the seventh rib. Complete parietal pleurectomy was performed, partial visceral pleurectomy was realized, only removing pleura invaded by malignant nodules (Figure 2). In some regions the disease resulted to be more infiltrating: three small atypical lung resections were necessary (Figure 3). On the basis of the cardiovascular comorbidity induced after HIPEC (atrial fibrillation), it was considered risky to subject the patient to HITHOC; it was decided to perform a pressurized intrathoracic aerosol chemotherapy (PITAC). It consists of a new way of performing intracavitary chemotherapy, more common than intraperitoneal use (PIPAC), but also promising for a pleural application (12).

On the basis of these considerations, following completion of cancer resection, the skin of the thoracotomy incision was sutured. Two trocars were introduced in the seventh and eighth intercostal spaces (Figure 4). The patient received PITAC using cisplatin at $7.5 \mathrm{mg} / \mathrm{sm}$ and doxorubicin at 1.5 $\mathrm{mg} / \mathrm{sm}$. The chemotherapy aerosol was generated at the tip of a mechanical micropump introduced through a balloon trocar and maintained for 30 minutes at $37^{\circ} \mathrm{C}$. Remotecontrolled aerosol nebulisation was performed in analogy to the PIPAC standard operating procedure. Then the toxic aerosol was exsufflated over a secure system into the outside environment. After completion of PITAC procedure two chest tubes were introduced using trocar accesses. During the dissection and PITAC procedure, the lung was maintained collapsed through the use of a double-lumen tube.

Postoperative daily chest radiographs showed no evidence of pulmonary edema. Postoperative course was uneventful and the patient was discharged on postoperative day 11. At approximately 6 months after the operation the patient remained without abdominal and thoracic recurrence.

\section{Results and Discussion}

Unlike most cancers, PMP rarely spreads through bloodstream or lymphatic vessels. Standard treatment for the abdominal disease is based on the combined approach of cytoreductive surgery and HIPEC. There is no standard treatment in case of intrathoracic involvement: pleural extension of PMP carries a poor prognosis and the current recommendation for the management of pleural-based disease is pleural cytoreduction combined with hyperthermic 


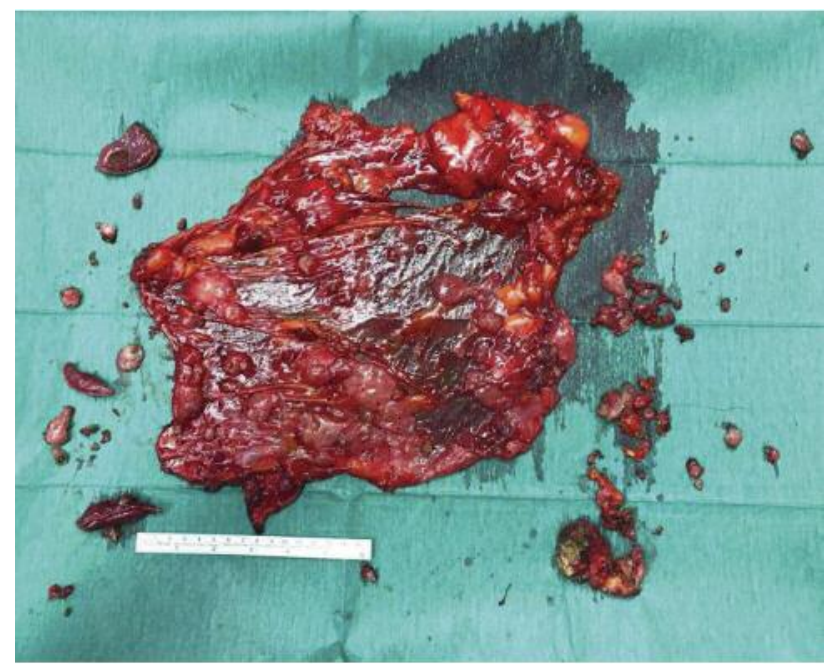

Figure 2. Complete removal of left parietal pleura. Small atypical pulmonary resections.

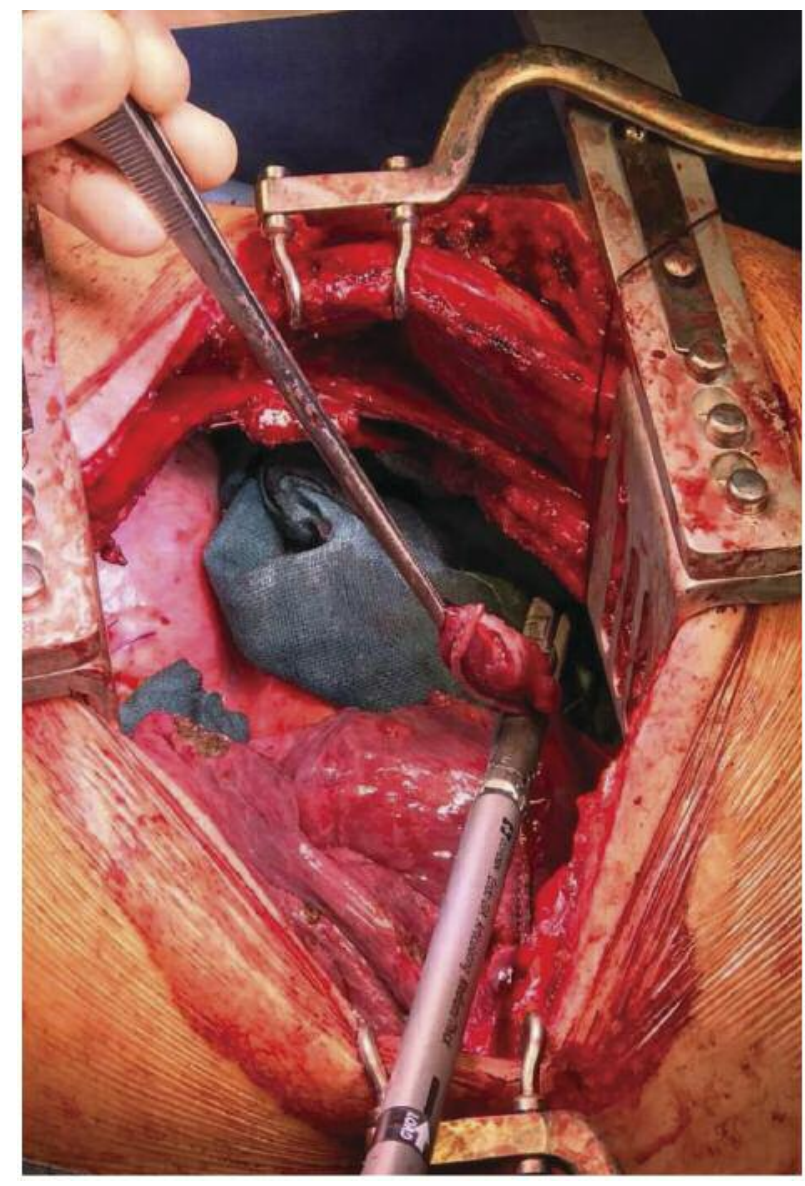

Figure 3. Pleurectomy and atypical lung resection.

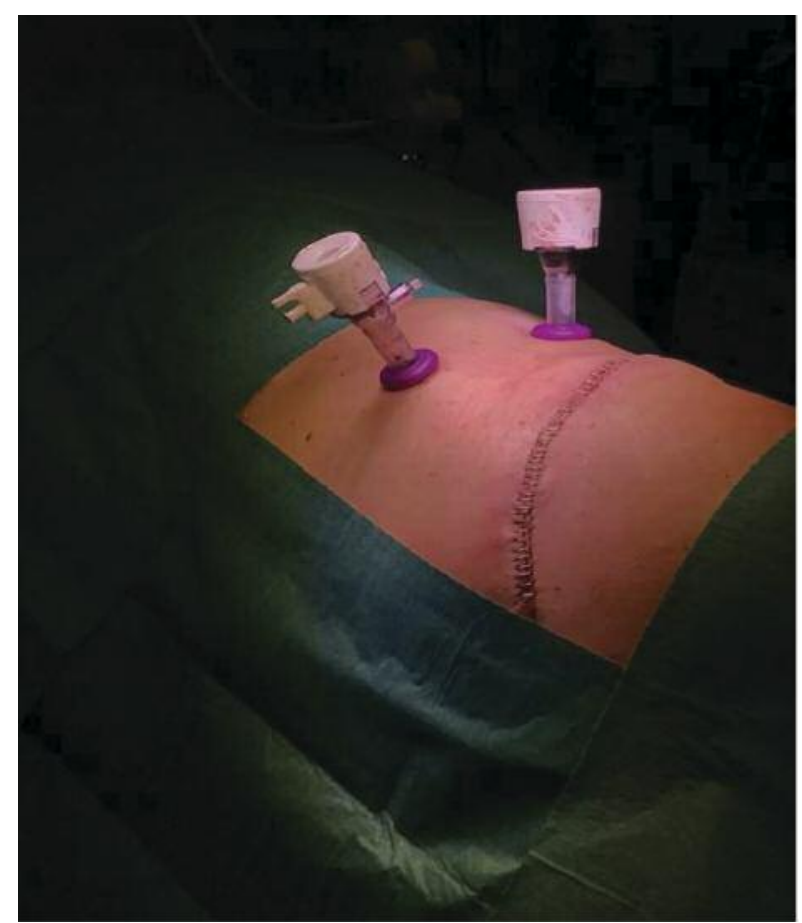

Figure 4. A two-trocar PITAC technique is used. The ipsilateral lung is excluded.

intrapleural chemotherapy; this kind of treatment is often associated with high morbidity and mortality. Because of the rarity of disease there are insufficient data even regarding the role of systemic chemotherapy, usually reserved to patients with unresectable disease.

According to available literature this is the first case report of a PMP with intrathoracic extension treated with pleurectomy, decortication and PITAC.

Intrapleural chemotherapy continues to be well used palliatively in order to control debilitating and unrelenting pleural effusions from cancer. Entrance into the tissues surrounding the thoracic cavity is by simple diffusion (13). A pharmacologic advantage of intracavitary chemotherapy administration within the thoracic space is reported in literature (14).

In this case, the goal of intrapleural chemotherapy application was a curative one. The administration was preceded by a thoracic cytoreduction. The aim of pleurectomy, decortication and lung resection was to remove all visible evidence of disease. The role of PITAC was to eliminate all microscopic residual disease.

The choice to perform PITAC instead of HITOC was made considering the patient's cardiological comorbidity. PIPAC, or abdominal administration of nebulized chemotherapy, is developing more and more. In our experience it turned out to be a well-tolerated procedure with no major complications. 
Pharmacokinetic and pharmacodynamic studies have also reported a higher tissue concentration than reported in HIPEC, using doses ten times lower.

To our knowledge, PITAC has been performed, to date, always in a palliative setting and has never been associated with cytoreductive surgery. In our experience, the association of pleurectomy and/or lung resection and PITAC is safe and feasible. No cardiovascular collapse nor pulmonary postoperative complications were observed. In these rare cases PITAC could be a good option. Further studies are needed to investigate the possible uses and role of this new procedure.

\section{References}

1 Geisinger KR, Levine EA, Shen $\mathrm{P}$ and Bradley RF. Pleuropulmonary involvement in pseudomyxoma peritonei: morphologic assessment and literature review. Am J Clin Pathol 127: 135-143, 2007.

2 Lee BY, Kim HS, Lee SH, Moon HS, Cho SM, Lee KH, Song KS, Min KO, Seo EJ and Lee JM. Pseudomyxoma peritonei: extraperitoneal spread to the pleural cavity and lung. J Thorac Imaging 19: 123-126, 2004.

3 Berge T: "Mucocele appendicis" with pseudomyxoma peritonei and pulmonary metastases. Acta Pathol Microbiol Scand 60: 483-486, 1964.

4 Kreissig P, Dancourt J and Garner G. Maladie gélatineuse du péritoine avec métastases pulmonaires [correspondence]. La Press Med 31: 1287, 1991.

5 Mortman KD, Sugarbaker PA, Shmookler BM, DeGuzman VC and Soberman MS: Pulmonary metastases in pseudomyxoma peritonei syndrome. Ann Thorac Surg 64: 1434-1436, 1997.

6 Pestieau SR, Wolk R and Sugarbaker PH: Congenital pleuroperitoneal communication in a patient with pseudomyxoma peritonei. J Surg Oncol 73: 174-178, 2000.

7 Pestieau SR, Esquivel J and Sugarbaker PH: Pleural extension of mucinous tumor in patients with pseudomyxoma peritonei syndrome. Ann Surg Oncol 7: 199-203, 2000.
8 Senthil M and Harrison LE: Simultaneous bicavitary hyperthermic chemoperfusion in the management of pseudomyxoma peritonei with synchronous pleural extension. Arch Surg 144: 970-972, 2009.

9 Ronnett BM, Zahn CM, Kurman RJ, Kass ME, Sugarbaker PH and Shmookler BM: Disseminated peritoneal adenomucinosis and peritoneal mucinous carcinomatosis: a clinicopathologic analysis of 109 cases with emphasis on distinguishing pathologic features, site of origin, prognosis and relationship to "pseudomyxoma peritonei”. Am J Surg Pathol 19: 1390-1408, 1995.

10 Carr NJ and Sobin LH: Tumors of the appendix. In: Bosman FT, Carneiro F, Hruban RH, Theise ND (Hrsg) WHO Classification of Tumors of the Digestive System 4. Aufl. World Health Organization Classification of Tumors, Bd 3. IARC Press, Lyon, France, S pp. 122-125, 2010.

11 De Simone M, Barone R, Vaira M, Aghemo B, Mioli P, Franco C, Scuderi S, Costamagna D and Dei Poli M: Semi-closed hyperthermic -antiblastic peritoneal perfusion (HAPP) in the treatment of peritoneal carcinosis. J Surg Oncol 82: 138-140, 2003.

12 Jonscher N, Hummel M, Giger-Pabst U, Karljalainen E, Zieren J, Büchner N, Reymond M and Beshay M: Pressurized intrathoracic aerosol chemotherapy (PITAC). In: Reymond M, Solass W (Hrsg), PIPAC - Pressurized IntraPeritoneal Aerosol Chemotherapy Cancer under Pressure. De Gruyter, 2014, pp. 183-194, 2014.

13 Dedrick RL and Flessner MF: Pharmacokinetic problems in peritoneal drug administration: tissue penetration and surface exposure. J Natl Cancer Inst 89: 480-487, 1997.

14 Sugarbaker PH, Stuart OA and Eger C: Pharmacokinetics of hyperthermic intrathoracic chemotherapy following pleurectomy and decortication. Gastroenterol Res Pract 2012: 471205, 2012.

Received November 7, 2017

Revised November 14, 2017

Accepted November 20, 2017 